\title{
A COMMENT ON THE LOW-DIMENSIONAL BUSEMANN-PETTY PROBLEM
}

\author{
EMANUEL MILMAN \\ DEPARTMENT OF MATHEMATICS, \\ THE WEIZMANN INSTITUTE OF SCIENCE, \\ REHOVOT 76100, ISRAEL. \\ E-MAIL: EMANUEL_MILMAN@HOTMAIL.COM.
}

\begin{abstract}
The generalized Busemann-Petty problem asks whether centrally-symmetric convex bodies having larger volume of all $m$ dimensional sections necessarily have larger volume. When $m>3$ this is known to be false, but the cases $m=2,3$ are still open. In those cases, it is shown that when the smaller body's radial function is a $n-m$-th root of the radial function of a convex body, the answer to the generalized Busemann-Petty problem is positive (for any larger star-body). Several immediate corollaries of this observation are also discussed.
\end{abstract}

\section{INTRODUCTION}

Let $\operatorname{Vol}(L)$ denote the Lebesgue measure of a set $L \subset \mathbb{R}^{n}$ in its affine hull, and let $G(n, k)$ denote the Grassmann manifold of $k$ dimensional subspaces of $\mathbb{R}^{n}$. Let $D_{n}$ denote the Euclidean unit ball, and $S^{n-1}$ the Euclidean sphere. All of the bodies considered in this note will be assumed to be centrally symmetric star-bodies, defined by a continuous radial function $\rho_{K}(\theta)=\max \{r \geq 0 \mid r \theta \in K\}$ for $\theta \in S^{n-1}$ and a starbody $K$.

The Busemann-Petty problem, first posed in [BP56, asks whether two centrally-symmetric convex bodies $K$ and $L$ in $\mathbb{R}^{n}$ satisfying:

$$
\operatorname{Vol}(K \cap H) \leq \operatorname{Vol}(L \cap H) \quad \forall H \in G(n, n-1)
$$

necessarily satisfy $\operatorname{Vol}(K) \leq \operatorname{Vol}(L)$. For a long time this was believed to be true (this is certainly true for $n=2$ ), until a first counterexample was given in LR75 for a large value of $n$. In the same year, the notion of an intersection-body was first introduced by Lutwak in [Lut75] (see also [ut88 and Section 2 for definitions) in connection to the Busemann-Petty problem. It was shown in [Lut88] (and refined in

Supported in part by BSF and ISF. 
Gar94a) that the answer to the Busemann-Petty problem is equivalent to whether all convex bodies in $\mathbb{R}^{n}$ are intersection bodies. Subsequently, it was shown in a series of results (LR75, Bal88, Bou91, Gia90, Pap92, Gar94a, Gar94c], Kol98b, Zha99, GKS99]), that this is true for $n \leq 4$, but false for $n \geq 5$.

In [Zha96], Zhang considered a natural generalization of the BusemannPetty problem, which asks whether two centrally-symmetric convex bodies $K$ and $L$ in $\mathbb{R}^{n}$ satisfying:

$$
\operatorname{Vol}(K \cap H) \leq \operatorname{Vol}(L \cap H) \forall H \in G(n, n-k)
$$

necessarily satisfy $\operatorname{Vol}(K) \leq \operatorname{Vol}(L)$, where $k$ is some integer between 1 and $n-1$. Zhang showed that the generalized $k$-codimensional Busemann-Petty problem is also naturally associated to another class of bodies, which will be referred to as $k$-Busemann-Petty bodies (note that these bodies are referred to as $n-k$-intersection bodies in Zha96] and generalized $k$-intersection bodies in [Kol00], and that the generalized $k$-codimensional problem is equivalent to whether all convex bodies in $R^{n}$ are $k$-Busemann-Petty bodies. Analogously to the original problem, it was shown in Zha96 that if $K$ and $L$ are two centrallysymmetric star-bodies (not necessarily convex) satisfying (1.2), and if $K$ is a $k$-Busemann-Petty body, then $\operatorname{Vol}(K) \leq \operatorname{Vol}(L)$.

It was shown in BZ98, and later in Kol00, that the answer to the generalized $k$-codimensional problem is negative for $k<n-3$, but the cases $k=n-3$ and $k=n-2$ still remain open (the case $k=n-1$ is obviously true). A partial answer to the case $k=n-2$ was given in BZ98, where it was shown that when $L$ is a Euclidean ball and $K$ is convex and sufficiently close to $L$, the answer is positive. Our main observation in this note concerns the cases $k=n-2, n-3$ and reads as follows:

Theorem 1.1. Let $K$ denote a centrally-symmetric convex body in $\mathbb{R}^{n}$. For $a=2,3$, let $K_{a}$ be the star-body defined by $\rho_{K_{a}}=\rho_{K}^{1 /(n-a)}$. Then $K_{a}$ is a $(n-a)$-Busemann-Petty body, implying a positive answer to the $(n-a)$-codimensional Busemann-Petty problem (1.2) for the pair $K_{a}, L$ for any star-body $L$.

The case $a=1$ is also true, but follows trivially since it is easy to see (e.g. [Mil05]) that any star-body is an $n-1$-Busemann-Petty body. The case $a=2$ follows from $a=3$ by a general result from Mil05, stating that if $K$ is a $k$-Busemann-Petty body and $L$ is given by $\rho_{L}=\rho_{K}^{k / l}$ for $1 \leq k<l \leq n-1$, then $L$ is a $l$-Busemann-Petty body.

Theorem 1.1 has several interesting consequences. The first one is the following complementary result to the one aforementioned from 
BZ98. Roughly speaking, it states that any small enough perturbation $K$ of the Euclidean ball, for which we have control over the second derivatives of $\rho_{K}$, satisfies the low-dimensional generalized BusemannPetty problem (1.2) with any star-body $L$.

Corollary 1.2. For any $n$, there exists a function $\gamma:[0, \infty) \rightarrow(0,1)$, such that the following holds: let $\varphi$ denote a twice continuously differentiable function on $S^{n-1}$ such that:

$$
\max _{\theta \in S^{n-1}}|\varphi(\theta)| \leq 1, \max _{\theta \in S^{n-1}}\left|\varphi_{i}(\theta)\right| \leq M, \max _{\theta \in S^{n-1}}\left|\varphi_{i, j}(\theta)\right| \leq M,
$$

for every $i, j=1, \ldots, n-1$, where $\varphi_{i}$ and $\varphi_{i, j}$ denote the first and second partial derivatives of $\varphi$ (w.r.t. any local coordinate system of $\left.S^{n-1}\right)$, respectively. Then the star-body $K^{\varepsilon}$ defined by $\rho_{K^{\varepsilon}}=1+\varepsilon \varphi$ for any $|\varepsilon|<\gamma(M)$ is a $(n-a)$-Busemann-Petty body for $a=2,3$, implying a positive answer to the $(n-a)$-codimensional Busemann-Petty problem (1.2) for $K^{\varepsilon}$ and any star-body $L$.

Note that the definition of $K_{a}$ in Theorem 1.1 is highly non-linear with respect to $K$. Since the class of $k$-Busemann-Petty bodies is closed under certain natural operations (see Mil05] for the latest known results), we can take advantage of this fact to strengthen the result of Theorem 1.1. For instance, it is well known (e.g. [GZ99, Mil05]) that the class of $k$-Busemann-Petty bodies is closed under taking $k$-radial sums. The $k$-radial sum of two star-bodies $L_{1}, L_{2}$ is defined as the star-body $L$ satisfying $\rho_{L}^{k}=\rho_{L_{1}}^{k}+\rho_{L_{2}}^{k}$. When $k=1$ this operation will simply be referred to as radial sum. The space of star-bodies in $\mathbb{R}^{n}$ is endowed with the natural radial metric $d_{r}$, defined as $d_{r}\left(L_{1}, L_{2}\right)=$ $\max _{\theta \in S^{n-1}}\left|\rho_{L_{1}}(\theta)-\rho_{L_{2}}(\theta)\right|$. We will denote by $\mathcal{R} \mathcal{C}^{n}$ the closure in the radial metric of the class of all star-bodies in $\mathbb{R}^{n}$ which are finite radial sums of centrally-symmetric convex bodies. It should then be clear that:

Corollary 1.3. Theorem 1.1 holds for any $K \in \mathcal{R C}^{n}$.

Our last remark in this note is again an immediate consequence of Theorem 1.1 and the following characterization of $k$-Busemann-Petty bodies due to Grinberg and Zhang ([GZ99]), which generalizes the characterization of intersection-bodies (the case $k=1$ ) given by Goodey and Weil (GW95]):

Theorem (Grinberg and Zhang). A star-body $K$ is a k-BusemannPetty body iff it is the limit of $\left\{K_{i}\right\}$ in the radial metric $d_{r}$, where each $K_{i}$ is a finite $k$-radial sums of ellipsoids $\left\{\mathcal{E}_{j}^{i}\right\}$ :

$$
\rho_{K_{i}}^{k}=\rho_{\mathcal{E}_{1}^{i}}^{k}+\ldots+\rho_{\mathcal{E}_{m_{i}}^{i}}^{k} .
$$


Applying Grinberg and Zhang's Theorem to the bodies $K_{a}$ from Theorem 1.1, we immediately have:

Corollary 1.4. Let $K$ denote a centrally-symmetric convex body in $\mathbb{R}^{n}$. Then for $a=2,3, K$ is the limit in the radial metric $d_{r}$ of star-bodies $K_{i}$ having the form:

$$
\rho_{K_{i}}=\rho_{\mathcal{E}_{1}^{i}}^{n-a}+\ldots+\rho_{\mathcal{E}_{m_{i}}^{i}}^{n-a},
$$

where $\left\{\mathcal{E}_{j}^{i}\right\}$ are ellipsoids.

\section{DEFINITIONS AND NOTATIONS}

A star body $K$ is said to be an intersection body of a star body $L$, if $\rho_{K}(\theta)=\operatorname{Vol}\left(L \cap \theta^{\perp}\right)$ for every $\theta \in S^{n-1} . K$ is said to be an intersection body, if it is the limit in the radial metric $d_{r}$ of intersection bodies $\left\{K_{i}\right\}$ of star bodies $\left\{L_{i}\right\}$, where $d_{r}\left(K_{1}, K_{2}\right)=\sup _{\theta \in S^{n-1}}\left|\rho_{K_{1}}(\theta)-\rho_{K_{2}}(\theta)\right|$. This is equivalent (e.g. [Lut88, Gar94a]) to $\rho_{K}=R^{*}(d \mu)$, where $\mu$ is a non-negative Borel measure on $S^{n-1}, R^{*}$ is the dual transform (as in (2.1) ) to the Spherical Radon Transform $R: C\left(S^{n-1}\right) \rightarrow C\left(S^{n-1}\right)$, which is defined for $f \in C\left(S^{n-1}\right)$ as:

$$
R(f)(\theta)=\int_{S^{n-1} \cap \theta^{\perp}} f(\xi) d \sigma_{n-1}(\xi)
$$

where $\sigma_{n-1}$ the Haar probability measure on $S^{n-2}$ (and we have identified $S^{n-2}$ with $\left.S^{n-1} \cap \theta^{\perp}\right)$.

Before defining the class of $k$-Busemann-Petty bodies we shall need to introduce the $m$-dimensional Spherical Radon Transform, acting on spaces of continuous functions as follows:

$$
\begin{array}{r}
R_{m}: C\left(S^{n-1}\right) \longrightarrow C(G(n, m)) \\
R_{m}(f)(E)=\int_{S^{n-1} \cap E} f(\theta) d \sigma_{m}(\theta),
\end{array}
$$

where $\sigma_{m}$ is the Haar probability measure on $S^{m-1}$ (and we have identified $S^{m-1}$ with $S^{n-1} \cap E$ ). The dual transform is defined on spaces of signed Borel measures $\mathcal{M}$ by:

$$
\begin{aligned}
& R_{m}^{*}: \mathcal{M}(G(n, m)) \longrightarrow \mathcal{M}\left(S^{n-1}\right) \\
& \int_{S^{n-1}} f R_{m}^{*}(d \mu)=\int_{G(n, m)} R_{m}(f) d \mu \quad \forall f \in C\left(S^{n-1}\right),
\end{aligned}
$$

and for a measure $\mu$ with continuous density $g$, the transform may be explicitly written in terms of $g$ (see [Zha96]):

$$
R_{m}^{*} g(\theta)=\int_{\theta \in E \in G(n, m)} g(E) d \nu_{m}(E),
$$


where $\nu_{m}$ is the Haar probability measure on $G(n-1, m-1)$.

We shall say that a body $K$ is a $k$-Busemann-Petty body if $\rho_{K}^{k}=$ $R_{n-k}^{*}(d \mu)$ as measures in $\mathcal{M}\left(S^{n-1}\right)$, where $\mu$ is a non-negative Borel measure on $G(n, n-k)$. We shall denote the class of such bodies by $\mathcal{B P}_{k}^{n}$. Choosing $k=1$, for which $G(n, n-1)$ is isometric to $S^{n-1} / Z_{2}$ by mapping $H$ to $S^{n-1} \cap H^{\perp}$, and noticing that $R$ is equivalent to $R_{n-1}$ under this map, we see that $\mathcal{B P}_{1}^{n}$ is exactly the class of intersection bodies.

We will also require, although indirectly, several notions regarding Fourier transforms of homogeneous distributions. We denote by $\mathcal{S}\left(\mathbb{R}^{n}\right)$ the space of rapidly decreasing infinitely differentiable test functions in $\mathbb{R}^{n}$, and by $\mathcal{S}^{\prime}\left(\mathbb{R}^{n}\right)$ the space of distributions over $\mathcal{S}\left(\mathbb{R}^{n}\right)$. The Fourier Transform $\hat{f}$ of a distribution $f \in \mathcal{S}^{\prime}\left(\mathbb{R}^{n}\right)$ is defined by $\langle\hat{f}, \phi\rangle=\langle f, \hat{\phi}\rangle$ for every test function $\phi$, where $\hat{\phi}(y)=\int \phi(x) \exp (-i\langle x, y\rangle) d x$. A distribution $f$ is called homogeneous of degree $p \in \mathbb{R}$ if $\langle f, \phi(\cdot / t)\rangle=$ $|t|^{n+p}\langle f, \phi\rangle$ for every $t>0$, and it is called even if the same is true for $t=-1$. An even distribution $f$ always satisfies $(\hat{f})^{\wedge}=(2 \pi)^{n} f$. The Fourier Transform of an even homogeneous distribution of degree $p$ is an even homogeneous distribution of degree $-n-p$.

We will denote the space of continuous functions on the sphere by $C\left(S^{n-1}\right)$. The spaces of even continuous and infinitely smooth functions will be denoted $C_{e}\left(S^{n-1}\right)$ and $C^{\infty}\left(S^{n-1}\right)$, respectively.

For a star-body $K$ (not necessarily convex), we define its Minkowski functional as $\|x\|_{K}=\min \{t \geq 0 \mid x / t \in K\}$. When $K$ is a centrallysymmetric convex body, this of course coincides with the natural norm associated with it. Obviously $\rho_{K}(\theta)=\|\theta\|_{K}^{-1}$ for $\theta \in S^{n-1}$.

\section{Proofs of the statements}

Before we begin, we shall need to recall several known facts about the Spherical Radon Transform $R$, and its connection to the Fourier transform of homogeneous distributions. It is well known (e.g. Gro96, Chapter 3]) that $R: C_{e}\left(S^{n-1}\right) \rightarrow C_{e}\left(S^{n-1}\right)$ is an injective operator, and that it is onto a dense set in $C_{e}\left(S^{n-1}\right)$ which contains $C_{e}^{\infty}\left(S^{n-1}\right)$. The connection with Fourier transforms of homogeneous distributions was demonstrated by Koldobsky, who showed (e.g. Kol98a]) the following:

Lemma 3.1. Let $L$ denote a star-body in $\mathbb{R}^{n}$. Then for all $\theta \in S^{n-1}$ :

$$
\left(\|\cdot\|_{L}^{-n+1}\right)^{\wedge}(\theta)=\pi(n-1) \operatorname{Vol}\left(D_{n-1}\right) R\left(\|\cdot\|_{L}^{-n+1}\right)(\theta) .
$$

In particular $\left(\|\cdot\|_{L}^{-n+1}\right)^{\wedge}$ is continuous, and of course homogeneous of degree -1 . Hence, if we denote $\rho_{K}(\theta)=\|\theta\|_{K}^{-1}=\left(\|\cdot\|_{L}^{-n+1}\right)^{\wedge}(\theta)$ for 
$\theta \in S^{n-1}$ and use $\left(\|\cdot\|_{K}^{-1}\right)^{\wedge}(\theta)=(2 \pi)^{n}\|\theta\|_{L}^{-n+1}$, we immediately get the following inversion formula for the Spherical Radon transform:

Lemma 3.2. Let $K$ denote a star-body in $\mathbb{R}^{n}$ such that $\rho_{K}$ is in the range of the Spherical Radon Transform. Then for all $\theta \in S^{n-1}$ :

$$
R^{-1}\left(\rho_{K}\right)(\theta)=\frac{\pi(n-1) \operatorname{Vol}\left(D_{n-1}\right)}{(2 \pi)^{n}}\left(\|\cdot\|_{K}^{-1}\right)^{\wedge}(\theta) .
$$

Koldobsky also discovered the following property of the Fourier transform of a norm of a convex body ([Kol00, Corollary 2]):

Lemma 3.3. Let $K$ be an infinitely smooth centrally-symmetric convex body in $\mathbb{R}^{n}$. Then for every $E \in G(n, k)$ :

$$
\int_{S^{n-1} \cap E}\left(\|\cdot\|_{K}^{-n+k+2}\right)^{\wedge}(\theta) d \theta \geq 0 .
$$

Since $C_{e}^{\infty}\left(S^{n-1}\right)$ is in the range of the Spherical Radon Transform, applying Lemma 3.3 with $k=n-3$ and using Lemma 3.2, we have:

Proposition 3.4. Let $K$ be an infinitely smooth centrally-symmetric convex body in $\mathbb{R}^{n}$. Then for every $E \in G(n, n-3)$ :

$$
\int_{S^{n-1} \cap E} R^{-1}\left(\rho_{K}\right)(\theta) d \theta \geq 0
$$

We are now ready to prove Theorem 1.1.

Proof of Theorem 1.1. First, assume that $K$ is infinitely smooth and fix $\theta \in S^{n-1}$. Denote by $H_{\theta} \in G(n, n-1)$ the hyperplane $\theta^{\perp}$, and let $\sigma_{H_{\theta}}$ denote the Haar probability measure on $S^{n-1} \cap H_{\theta}$. Let $\eta_{H_{\theta}}$ denote the Haar probability measure on the homogeneous space $G^{H_{\theta}}(n, n-3):=$ $\left\{E \in G(n, n-3) \mid E \subset H_{\theta}\right\}$, and let $\sigma_{E}$ denote the Haar probability measure on $S^{n-1} \cap E$ for $E \in G(n, n-3)$. Then:

$$
\begin{array}{r}
\rho_{K}(\theta)=R\left(R^{-1}\left(\rho_{K}\right)\right)(\theta)=\int_{S^{n-1} \cap H_{\theta}} R^{-1}\left(\rho_{K}\right)(\xi) d \sigma_{H_{\theta}}(\xi) \\
=\int_{E \in G^{H_{\theta}(n, n-3)}} \int_{S^{n-1} \cap E} R^{-1}\left(\rho_{K}\right)(\xi) d \sigma_{E}(\xi) d \eta_{H_{\theta}}(E) .
\end{array}
$$

The last transition is explained by the fact that the measure $d \sigma_{E}(\xi) d \eta_{H_{\theta}}(E)$ is invariant under orthogonal transformations preserving $H_{\theta}$, so by the uniqueness of the Haar probability measure, it must coincide with $d \sigma_{H_{\theta}}(\xi)$. Denoting:

$$
g(F)=\int_{S^{n-1} \cap F^{\perp}} R^{-1}\left(\rho_{K}\right)(\xi) d \sigma_{E}(\xi)
$$


for $F \in G(n, 3)$, we see by Proposition 3.4 that $g \geq 0$. Plugging the definition of $g$ in (3.1), we have:

$$
\rho_{K}(\theta)=\int_{E \in G^{H_{\theta}(n, n-3)}} g\left(E^{\perp}\right) d \eta_{H_{\theta}}(E)=\int_{F \in G_{\theta}(n, 3)} g(F) d \nu_{\theta}(F),
$$

where $\nu_{\theta}$ is the Haar probability measure on the homogeneous space $G_{\theta}(n, 3):=\{F \in G(n, 3) \mid \theta \in F\}$ and the transition is justified as above. By (2.2), we conclude that $\rho_{K}=R_{3}^{*}(g)$ with $g \geq 0$, implying that the body $K_{3}$ satisfying $\rho_{K_{3}}^{n-3}=\rho_{K}$ is in $\mathcal{B P}_{n-3}^{n}$.

As mentioned in the Introduction, the case $a=2$ follows from $a=3$ by a general result from [Mil05], but for completeness we reproduce the easy argument. Using double-integration as before:

$\rho_{K}(\theta)=\int_{F \in G_{\theta}(n, 3)} g(F) d \nu_{\theta}(F)=\int_{J \in G_{\theta}(n, 2)} \int_{F \in G_{J}(n, 3)} g(F) d \nu_{J}(F) d \mu_{\theta}(J)$,

where $\mu_{\theta}$ and $\nu_{J}$ are the Haar probability measures on the homogeneous spaces $G_{\theta}(n, 2):=\{J \in G(n, 2) \mid \theta \in J\}$ and $G_{J}(n, 3):=\{F \in G(n, 3) \mid J \subset F\}$, respectively. Denoting:

$$
h(J)=\int_{F \in G_{J}(n, 3)} g(F) d \nu_{J}(F),
$$

we see that $h \geq 0$ and $\rho_{K}=R_{2}^{*}(h)$, implying that the body $K_{2}$ satisfying $\rho_{K_{2}}^{n-2}=\rho_{K}$ is in $\mathcal{B P}_{n-2}^{n}$.

When $K$ is a general convex body, the result follows by approximation. It is well known (e.g. SSch93, Theorem 3.3.1]) that any centrallysymmetric convex body $K$ may be approximated (for instance in the radial metric) by a series of infinitely smooth centrally-symmetric convex bodies $\left\{K^{i}\right\}$. Denoting by $K_{a}^{i}$ the star-bodies satisfying $\rho_{K_{a}^{i}}=\rho_{K_{i}}^{1 /(n-a)}$ for $a=2,3$, we have seen that $K_{a}^{i} \in \mathcal{B P}_{n-a}^{n}$. Obviously the series $\left\{K_{a}^{i}\right\}$ tends to $K_{a}$ in the radial metric, and since $\mathcal{B P}_{n-a}^{n}$ is closed under taking radial limit (see Mil05]), the result follows.

We now turn to close a few loose ends in the proof of Corollary 1.3. Since $\mathcal{B P}_{k}^{n}$ is closed under $k$-radial sums, it is immediate that if $K^{1}$ and $K^{2}$ are two convex bodies, $L$ is their radial sum, and $\rho_{T_{a}}=\rho_{T}^{1 /(n-a)}$ for $T=K_{1}, K_{2}, L$, then:

$$
\rho_{L_{a}}^{n-a}=\rho_{L}=\rho_{K_{1}}+\rho_{K_{2}}=\rho_{K_{a}^{1}}^{n-a}+\rho_{K_{a}^{2}}^{n-a},
$$

and therefore $L_{a} \in \mathcal{B P}_{n-a}^{n}$. This argument of course extends to any finite radial sum of convex bodies, and since $\mathcal{B P}_{k}^{n}$ is closed under taking limit in the radial metric, the argument extends to the entire class $\mathcal{R C}^{n}$ defined in the Introduction. 
It remains to prove Corollary 1.2 .

Proof of Corollary 1.2. By Theorem 1.1, it is enough to show that for a small enough $|\varepsilon|$ (which depends on $n$ and $M$ ), the star-bodies $L_{a}^{\varepsilon}$ defined by $\rho_{L_{a}^{\varepsilon}}=\rho_{K^{\varepsilon}}^{n-a}$ are in fact convex. Since $\rho_{L_{a}^{\varepsilon}}=(1+\varepsilon \varphi)^{n-a}$, it is clear that for every $\theta \in S^{n-1}$ :

$\left|\rho_{L_{a}^{\varepsilon}}(\theta)\right| \leq f_{0}(\varepsilon, n),\left|\left(\rho_{L_{a}^{\varepsilon}}\right)_{i}(\theta)\right| \leq f_{1}(\varepsilon, n, M),\left|\left(\rho_{L_{a}^{\varepsilon}}\right)_{i, j}(\theta)\right| \leq f_{2}(\varepsilon, n, M)$,

for every $i, j=1, \ldots, n-1$, where $f_{0}$ tends to 1 and $f_{1}, f_{2}$ tend to 0 , as $\varepsilon \rightarrow 0$. It should be intuitively clear that the convexity of $L_{a}^{\varepsilon}$ depends only on the behaviour of the derivatives of order 0,1 and 2 of $\rho_{L_{a}^{\varepsilon}}$, and since we have uniform convergence of these derivatives to those of the Euclidean ball as $\varepsilon$ tends to $0, L_{a}^{\varepsilon}$ is convex for small enough $\varepsilon$. To make this argument formal, we follow Gar94b, and use a formula for the Gaussian curvature of a star-body $L$ whole radial function $\rho_{L}$ is twice continuously differentiable, which was explicitly calculated in Oli84, 2.5]. In particular, it follows that $M_{L}(\theta)$, the Gaussian curvature of $\partial L$ (the hypersurface given by the boundary of $L$ ) at $\rho_{L}(\theta) \theta$, is a continuous function of the derivatives of order 0,1 and 2 of $\rho_{L}$ at the point $\theta$. Since the Gaussian curvature of the boundary of the Euclidean ball is a constant 1, it follows that for small enough $\varepsilon$, the boundary of $L_{a}^{\varepsilon}$ has everywhere positive Gaussian curvature. By a standard result in differential geometry (e.g. [KN69, p. 41]), this implies that $L_{a}^{\varepsilon}$ is convex. This concludes the proof.

\section{REFERENCES}

[Bal88] K. Ball, Some remarks on the geometry of convex sets, Lecture Notes in Mathematics, vol. 1317, Springer-Verlag, 1988, pp. 224-231.

[Bou91] J. Bourgain, On the busemann-petty problem for perturbations of the ball, Geometric and Functional Analysis 1 (1991), 1-13.

[BP56] H. Busemann and C.M. Petty, Problems on convex bodies, Math. Scand. 4 (1956), 88-94.

[BZ98] J. Bourgain and G. Zhang, On a generalization of the busemann-petty problem, Convex Geometric Analysis 34 (1998), 65-76.

[Gar94a] R.J. Gardner, Intersection bodies and the busemann-petty problem, Transactions of the American Mathematical Society 342 (1994), no. 1, 435-445.

[Gar94b] R.J. Gardner, Intersection bodies and the busemann-petty problem, Transactions of the American Mathematical Society 342 (1994), no. 1, 435-445.

[Gar94c] R.J. Gardner, A positive answer to the busemann-petty problem in three dimensions, Annals of Mathematics 140 (1994), 435-447.

[Gia90] A.A. Giannopoulos, A note on a problem of h. busemann and c.m. petty concerning sections of symmetric convex bodies, Mathematika 37 (1990), 239-244. 
[GKS99] R.J. Gardner, A. Koldobsky, and Th. Schlumprecht, An analytic solution to the busemann-petty problem on sections of convex bodies, Annals of Mathematics 149 (1999), 691-703.

[Gro96] H. Groemer, Geometric applications of fourier series and spherical harmonics, Encyclopedia of Mathematics and its Applications, vol. 61, Cambridge University Press, New-York, 1996.

[GW95] P. Goodey and W. Weil, Intersection bodies and ellipsoids, Mathematika 42 (1995), 295-304.

[GZ99] E.L. Grinberg and G. Zhang, Convolutions, transforms, and convex bodies, Proc. London Math. Soc. 78 (1999), no. 3, 77-115.

[KN69] S. Kobayashi and K. Nomizu, , Interscience Tracts in Pure and Applied Mathematics, No. 15 Vol. II, Interscience Publishers John Wiley \& Sons, Inc., New York-London-Sydney, 1969.

[Kol98a] A. Koldobsky, An application of the fourier transform to sections of star bodies, Israel Journal of Mathematics 106 (1998), 157-164.

[Kol98b] A. Koldobsky, Intersection bodies in $\mathbb{R}^{4}$, Advances in Mathematics 136 (1998), 1-14.

[Kol00] A. Koldobsky, A functional analytic approach to intersection bodies, Geometric and Functional Analysis 10 (2000), 1507-1526.

[LR75] D.G. Larman and C.A. Rogers, The existence of a centrally symmetric convex body with central sections that are unexpectedly small, Mathematika 22 (1975), 164-175.

[Lut75] E. Lutwak, Dual mixed volumes, Pacific Journal of Mathematics 58 (1975), 531-538.

[Lut88] E. Lutwak, Intersection bodies and dual mixed volumes, Advances in Mathematics 71 (1988), 232-261.

[Mil05] E. Milman, Generalized intersection bodies, Manuscript, 2005.

[Oli84] V. I. Oliker, Hypersurfaces in $\mathbf{R}^{n+1}$ with prescribed Gaussian curvature and related equations of Monge-Ampère type, Comm. Partial Differential Equations 9 (1984), no. 8, 807-838.

[Pap92] M. Papadimitrakis, On the busemann-petty problem about convex, centrally symmetric bodies in $\mathbb{R}^{n}$, Mathematika 39 (1992), 258-266.

[Sch93] R. Schneider, Convex bodies: the Brunn-Minkowski theory, Encyclopedia of Mathematics and its Applications, vol. 44, Cambridge University Press, Cambridge, 1993.

[Zha96] G. Zhang, Sections of convex bodies, American Journal of Mathematics 118 (1996), 319-340.

[Zha99] G. Zhang, A positive solution to the busemann-petty problem in $\mathbf{r}^{4}$, Ann. of Math. 149 (1999), no. 2, 535-543. 